This is the final peer-reviewed accepted manuscript of:

Fino, E., Menegatti, M., Avenanti, A., \& Rubini, M. (2016). Enjoying vs. smiling: Facial muscular activation in response to emotional language. Biological psychology, 118, 126-135.

The final published version is available online at: https://doi.org/10.1016/j.biopsycho.2016.04.069

Rights / License:

The terms and conditions for the reuse of this version of the manuscript are specified in the publishing policy. For all terms of use and more information see the publisher's website. 
RUNNING HEAD: Facial responses to emotional language

\title{
Enjoying vs. smiling: Facial muscular activation in response to emotional language
}

\author{
Edita Fino, Michela Menegatti, Alessio Avenanti, and Monica Rubini
}

Edita Fino, Department of Sociology, Psychology and Education, Social Brain Lab (SBL), University Marin Barleti, Rr. Sami Frasheri, 41, 1000, Tirana, Albania; sbl@umb.edu.al Michela Menegatti, Department of Psychology, Alma Mater Studiorum Università di Bologna, Viale Berti Pichat 5, 40126, Bologna, Italy; m.menegatti@unibo.it

Alessio Avenanti, Department of Psychology, Centre for studies and research in Cognitive Neuroscience (CsrNC), Alma Mater Studiorum Università di Bologna, Viale Europa 980, 47023, Cesena, Italy, and IRCCS Fondazione Santa Lucia, Roma, Italy; alessio.avenanti@unibo.it

Monica Rubini, Department of Psychology, Social Psychology Lab, Alma Mater Studiorum Università di Bologna, Viale Berti Pichat 5, 40126, Bologna, Italy; monica.rubini@unibo.it.

Correspondence concerning this article should be addressed to:

Edita Fino, Department of Sociology, Psychology and Education, Social Brain Lab, University Marin Barleti, Rr. Sami Frasheri, 41, 1000, Tirana, Albania; sbl@umb.edu.al 


\title{
Enjoying vs. smiling: Facial muscular responses to emotional language
}

\begin{abstract}
The present study examined whether emotionally congruent facial muscular activation - a somatic index of emotional language embodiment can be elicited by reading subject-verb sentences composed of action verbs, that refer directly to facial expressions (e.g., Mario smiles), but also by reading more abstract state verbs, which provide more direct access to the emotions felt by the agent (e.g., Mario enjoys). To address this issue, we measured facial electromyography (EMG) while participants evaluated state and action verbs sentences. We found emotional sentences including both verb categories to have valence-congruent effects on emotional ratings and corresponding facial muscle activations. As expected, state verbsentences were judged with higher valence ratings than action verbs-sentences. Moreover, despite emotional congruent facial activations were similar for the two linguistic categories, in a late temporal window we found a tendency for greater EMG modulation when reading action relative to state verbs sentences. These results support embodied theories of language comprehension and suggest that understanding emotional action and state verb sentences relies on partially dissociable motor and emotional processes.
\end{abstract}

Keywords: EMG, emotion language, linguistic abstraction, embodied cognition, zygomaticus, corrugator 
Seeing a face smiling or frowning automatically elicits corresponding facial expressions in the observer and this effect appears largely automatic and unconscious (Chartrand \& Van Baaren, 2009; Dimberg \& Petterson, 2000; Dimberg, Thunberg, \& Elmehed, 2000). The same facial reactions have been observed during reading of verbs referring directly to facial expressions, such as 'to smile' or 'to frown' (Foroni \& Semin, 2009). These effects have been explained as indices of the bodily grounding of language by embodied cognition theories, according to which language comprehension is not amodal as traditionally assumed, but involves a mental simulation grounded in bodily and neural states associated with the real world experience of perception, action or emotion (Barsalou, 1999; Glenberg, 2007; Niedenthal, Winkielman, Mondillon, \& Vermeulen, 2009; Niedenthal, Mermillod, Maringer, \& Hess, 2010). By simulation, such theories generally mean a representation of the situations, objects or events described in the text that is instantiated in the same neural systems used in original experience. Thus, simulation is implemented in multimodal brain areas that correspond to the referents in the language and plays a role in grasping the language meaning.

Accordingly, the comprehension of words or sentences conveying emotional meaning requires a partial reactivation of (embodied) sensorimotor and emotional states associated with the emotion experience described in the language (e.g., Barsalou, 1999; Barsalou, Niedenthal, Barbey, \& Ruppert, 2003; Barrett, 2006; Niedenthal et al., 2009; 2010). A body of research has focused in particular on overt bodily manifestations of such (covert) embodied simulation of language meanings. This research has addressed the peripheral motor correlates of language comprehension using facial electromyography (EMG), that is by investigating how understanding negative and positive verbal stimuli affects the activity of the corrugator supercilii (i.e., the brow muscle, which is involved in pulling the brow down and together into a frown) and the zygomaticus major (i.e., the cheek muscle, which pulls the 
corners of the mouth back and up into a smile). This research has built upon classical studies showing that verbal descriptions that elicit negative emotions activate the corrugator, whereas positive verbal material activates the zygomaticus (e.g., Vrana, 1993; Vrana \& Rollok, 2002; Larsen, Norris, \& Cacioppo, 2003) and indicating that the magnitude of emotional congruent facial activation is stronger for more emotionally valenced stimuli (Larsen et al., 2003).

Relevant to the embodied cognition framework, emotion congruent facial reactions were detected even when processing single words referring to emotion concepts. Niedenthal et al. (2009) asked participants to read emotional words referring to concrete objects (e.g., "cadaver", "gift" or "table") or abstract characteristics such as adjectives (e.g., "joyful", "repelled", "irritable" or "substantive"). Participants had to either judge whether each word was associated with an emotion (semantic task) or to simply indicate whether the word was written in lower-or uppercase letters (perceptual task). The results showed that zygomaticus activity was greater when participants processed words related to joy, whereas corrugator activity was greater for words related to anger and disgust. Interestingly, these effects occurred only in the semantic and not in the perceptual task. From these studies, it was concluded that emotional words are likely to induce emotionally congruent facial reactions a somatic marker of emotion embodiment - putatively as a result of activation of emotional and motor programs involved in the experience of referred emotions. Such emotion-related re-enactment is particularly relevant when participants are required to process word meaning, no matter if the word is concrete or abstract.

While a large corpus of evidence has been collected on the effect of emotional language (e.g., emotional sentences; single words) in triggering emotionally congruent facial activations, less is known about the role of linguistic categories at different levels of abstraction in eliciting such responses. The present research investigated this issue by comparing automatic facial muscular reactions to sentences composed of verbs referring to 
concrete actions (e.g., "Mario smiles") and those referring to emotional states (e.g., "Mario enjoys") which are more abstract (Semin \& Fiedler, 1988).

Relevant to the present research, a number of scholars have addressed the role of motor activations in understanding action verbs (de Vega, Glenberg, \& Graesser, 2008; Glenberg, 2007; Pulvermüller, 1999, 2005; Vigliocco, Vinson, Drucks, Barber, \& Cappa, 2011). In this account, processing language referring directly to an action also involves a simulation of the meaning that is instantiated (at least partially) in the motor system. Thus, reading a description of a facial action (i.e., to smile) maps directly onto motor programs responsible for that action and this could result in a subliminal activation of corresponding motor responses (i.e., a smiling response). This was recently demonstrated by Foroni and Semin (2009) who examined the effect of reading concrete and abstract words semantically associated with facial expressions of emotion on facial muscle activation. Participants read action verbs in the infinite form referring directly to facial expressions, such as "to smile" or "to frown", which are very concrete, and adjectives expressing emotional states (e.g., "funny" or "irritating") that are more abstract and do not directly refer to specific facial movements. Results showed that reading concrete action verbs 'to smile' and 'to frown' significantly activated the corresponding facial muscles (i.e., the zygomaticus and corrugator, respectively). Similar effects were also found for abstract adjectives, although in this case facial muscle activity was weaker in intensity. Similar findings were reported in a subsequent study (Foroni \& Semin, 2013) in which subject-verb sentences in the first person perspective (e.g., "I am smiling") were used.

However, these works have left unanswered the question of how processing sentences composed of verb categories encoded at different levels of abstraction, specifically abstract verbs referring to emotional states (e.g., to enjoy or to enrage) relative to concrete action verbs that refer to facial actions per se (e.g., to smile, or to frown) activates facial muscles. 
Indeed, Foroni and Semin $(2009 ; 2013)$ limited their focus to descriptive action verbs (e.g., to smile) and adjectives and did not consider that the linguistic repertoire through which emotions are expressed comprises another important and frequently used verb category, namely state verbs, which refers directly to felt emotions (e.g., surprised, amazed, angry; Semin \& Fiedler, 1991).

The present study aimed to fill this gap by examining whether somatic indices of emotion/motor simulation - i.e., emotionally congruent facial muscular activation - can be elicited not only by reading action verbs that refer directly to facial expressions (e.g., to smile, to frown), but also by reading state verbs, which have an indirect reference to facial expressions (e.g., to enjoy, to enrage may be associated with facial expressions of smiling and frowning) and are encoded at a higher level of abstraction (Coenen, Hedebouw \& Semin, 2006; Semin \& Fiedler, 1991).

We asked participants to read and evaluate subject-verb sentences including emotional action verbs (e.g., Mario smiles) and state verbs (e.g., Mario enjoys) while recording EMG from the zygomaticus and corrugator muscles. According to the Linguistic Category Model, state verbs, compared to descriptive action verbs, are more abstract and thus may generalize across different situations and observable behaviors (Semin \& Fiedler, 1988; 1991). Interestingly, higher levels of language abstraction implicitly convey more information about the subject and less about the specific situation. This means that state verbs provide stronger reference to the emotions felt by the agent in the sentence (see, Rubini, Menegatti, \& Moscatelli, 2014; Semin \& Marsman, 1994) and thus are expected to elicit stronger emotions relative to descriptive action verbs. Therefore, comparing facial muscular reactions and emotional ratings when reading action and state verbs allows for a more stringent testing of the effect of linguistic abstraction and emotionality on an established marker of emotion embodiment (i.e., emotion congruent facial activations) and emotion perception within two 
verb categories at different levels of linguistic abstractness.

According to the embodied cognition framework which suggests a simulation of the linguistic meaning, we assume that both sentences including state verbs and descriptive action verbs would trigger a convergence of emotion and motor simulation in the reader. Thus, both linguistic categories are expected to be perceived as emotional sentences and induce robust emotionally congruent facial activations. More specifically, we expect that reading sentences with emotionally positive meanings will be associated to positive ratings and the activation of the zygomaticus, whereas reading sentences with emotionally negative meanings will be associated to negative ratings and the activation of the corrugator.

However, we also expect a partial double dissociation for the two linguistic categories in behavioral ratings and facial EMG. Because state verbs convey more emotional information about the feeling agent (Menegatti \& Rubini, 2014; Rubini, Menegatti, \& Moscatelli, 2014; Semin \& Marsman, 1994), we expect that sentences including these verbs would be perceived as more emotional by participants relative to those including action verbs. On the other hand, based on the previous work of Foroni and Semin $(2009,2013)$ and the notion that action language directly maps onto motor responses (de Vega et al., 2008; Glenberg \& Gallese, 2012; Pulvermüller, 1999, 2005; Vigliocco et al., 2011), we hypothesized that understanding action sentences would result in a relatively high emotionally congruent facial activation - although we maintain this superiority to be relatively weak in view of the intrinsically lower emotional feature of action as compared to state verbs.

\section{Method}

\section{Participants}

Twenty undergraduate students of the University of Bologna (17 females, 3 males; mean age 22 years old) volunteered to participate in the experiment for academic credit. All 
subjects had normal or corrected-to-normal vision, were right-handed and were ignorant of the real purpose of the experiment. Prior to the start of the experiment, they read the ethical approval statement and filled out the informed consent form.

\section{Linguistic Stimuli}

The stimulus materials consisted in 12 Italian verbs ( 6 descriptive action verbs and 6 state verbs) related to positive and negative emotion expressions and 6 neutral fillers. Verbs were embedded into subject-verb sentences. Verbs were presented in the present tense and attributed to a third-person unknown agent (e.g., "Mario smiles"). For positive expressions of emotions, we used descriptive action verbs to smile (Mario sorride), to laugh (Mario ride), and to grin (Mario ridacchia) which directly relate to cheek actions and state verbs to enjoy (Mario gioisce), to be excited (Mario si rallegra), and be enthused (Mario si entusiasma) which refer to more abstract positive emotional states. For negative emotional expressions, we used descriptive action verbs to frown (Mario corruccia), scowl (Mario aggrotta) and glare (Mario si acciglia) which refer to brow actions and state verbs: get angry (Mario si arrabbia), infuriate (Mario si infuria), and irritate (Mario si irrita) which refer to more abstract negative emotional states.

We also included filler terms that refer to emotionally neutral actions that is, without any emotional load. Specifically, since state verbs always retain an emotional facet, we used concrete descriptive action verbs to walk, (Mario passeggia), to travel (Mario viaggia), to work (Mario lavora), which refer to very specific behaviors, and more abstract interpretative action verbs to try (Mario cerca), to change (Mario cambia), to introduce her/himself (Mario si presenta), which refer to broader classes of behaviors. Please note that filler terms do not retain any reference to the bodily parts involved in the experiment (i.e., facial muscles). English translations in some cases are approximate, as no precise correspondence is available. In the preparation of our stimuli we were limited by the aim of the study being that of 
examining facial responses to sentences resembling natural language that might not perfectly match in variables such as length or frequency in language use. However, we did not find any significant differences between stimulus categories in terms of length and frequency ${ }^{1}$. In particular, we found no effect of linguistic category or valence when considering our critical conditions namely positive and negative verbs.

\section{Procedure, Apparatus, and Data Acquisition}

Participants were asked to participate in a study on language reading and were tested individually. They were asked to read and evaluate a series of verbal stimuli presented on a computer using a likability scale. In order to shift attention from the electrodes and possible reference to facial muscle activity participants were told that the study concerned measuring skin conductance levels during word reading which would be recorded via sensors placed on the face (e.g., Dimberg et al., 2000). The EMG electrodes were placed on the zygomaticus and corrugator muscles of the left side of the face based on research showing higher activity levels of left versus right side facial musculature (Dimberg \& Petterson, 2000). The computer task consisted in sequential presentation of verbal material on a monitor and subsequent evaluation. In order for participants to familiarize with the computer task 10 practice trials were administered prior to the start of the experiment. During the experiment, the verbal stimuli were presented in a random order with E-prime software (Psychology Software Tools, Pittsburgh, PA) on a monitor located approximately $1 \mathrm{~m}$ from the participant. Each trial was composed of a central fixation cross (duration of $2000 \mathrm{~ms}$ ), followed by the presentation of the target subject-verb sentence (duration of $3000 \mathrm{~ms}$ ), an evaluation scale (average duration

\footnotetext{
${ }^{1}$ In order to check whether our stimulus material was matched for word length we conducted two a 2 (Valence: positive, negative) $\times 2$ (Linguistic category: descriptive action verb, state action verb) repeated measures ANOVAs on word length in terms of number of letters. The analysis was not significant $(F s<2.38$, ps $>.16)$. For word frequency, we analyzed the arcsine-transformed frequencies of use of the stimulus material in the Italian language (ColFis corpora; Bertinetto, Burani, Laudanna, Marconi, Ratti, Rolando, \& Thornton, 2005) using a similar Valence $\times$ Linguistic category ANOVA. Also this analysis provided no significant effect was found $(F \mathrm{~s}<3.47, p \mathrm{~s}>.1)$.
} 
$\sim 4$ seconds) and blank screen (1 second). Thus, the inter-stimulus interval was $\sim 10$ seconds allowing sufficient time for facial muscles to relax after stimulus presentation.

Participants were instructed to evaluate each stimulus sentence by right hand clicking on a 5 point Likert scale from 1 (I don't like it at all) to 5 (I like it very much). The likeability scale appeared on the monitor after each stimulus sentence and disappeared upon subjects' response. Participants were instructed to provide their response about 2-3 seconds after the presentation of the evaluation scale (mean RT was $\sim 4$ seconds) to minimize the interferential effect of hand motor response on facial EMG acquired during stimulus presentation.

The liking task was included to ensure that participants processed the emotional content of target stimuli. This was done in order to maximize the chance of detecting robust emotionally congruent face reactions, based on the evidence that active emotion evaluation of verbal material is associated to stronger facial reactivity (Niedenthal et al., 2009) and pleasantness evaluation is associated to earlier facial reactivity (Lanctot \& Hess, 2007). For each participant three blocks of stimuli were presented with each block containing all of the 12 verbs referring to emotional expressions and 6 neutral fillers in random order, resulting in 72 trials altogether.

During subject-verb presentation, facial muscle activity was measured using miniature $\mathrm{Ag} / \mathrm{AgCl}$ surface electrodes (4mm) attached over the left corrugator (brow) and zygomaticus (cheek) muscle regions according to the guidelines established by Fridlund and Cacioppo (1986). The skin was cleaned with disposable pads (70\% alcohol and pumice) and prepared for electrode placement to reduce skin impedance to less than $10 \mathrm{k} \Omega$. The raw EMG activity was measured with Biopack Systems MP36 data acquisition unit at a sampling rate of 1000 $\mathrm{Hz}$ using two bipolar channels and a gain of 1,000. The EMG signal was pass filtered online with a $20-250 \mathrm{~Hz}$ passband and a $50 \mathrm{~Hz}$ notch filter and was full-wave rectified offline. After the task, participants completed a manipulation check and filled out a questionnaire in which 
they were asked about their ideas regarding the purpose of the experiment. No one of the participants was aware of the hypotheses, and none suspected that facial muscular reactions were being measured. They were then debriefed and dismissed.

\section{Dependent variables}

\section{Manipulation checks}

To check whether positive and negative subject-verb sentences were evaluated as positive and negative, respectively, participants were asked to assess the valence of each sentence on a 7-point Likert scale from 1 (very positive) to 7 (very negative) in a separate session upon completion of the experimental task. Mean ratings of stimulus valence were entered in a 2 (Valence: positive, negative) $\times 2$ (Linguistic category: descriptive action verb, state verb) repeated measures ANOVA. Data from 2 participants were excluded from analyses due to missing values ${ }^{2}$. Post-hoc analysis was carried out with Duncan test to correct for multiple comparisons.

\section{Evaluative ratings}

Liking ratings of subject-verb sentences were provided after stimulus presentation by right-hand clicking on a 5-point Likert scale from 1 (I don't like it at all) to 5 (I like it very much). Mean rating values were analyzed with a 2 (Valence: positive, negative) $\times 2$ (Linguistic category: descriptive action verb, state verb) repeated measures ANOVA. Data from 6 participants were excluded from the analysis due to technical failure ${ }^{3}$. Post-hoc analysis was carried out with Duncan test to correct for multiple comparisons.

\footnotetext{
${ }^{2}$ Two participants did not complete post-experimental valence ratings. We decided to exclude those two participants from the analysis of valence but keep them in the facial EMG analysis considering that valence was measured in a separate session and analyzed independently from the other dependent variables. In this way, the sample size for our EMG analysis $(N=20)$ remained comparable to that of previous research exploring emotionally congruent facial reactions (see, Foroni \& Semin, 2013; Korb, Grandjean, \& Scherer, 2010; Delplanque et al., 2009; Vrana \& Gross, 2004).

${ }^{3}$ In six participants responses on the evaluation task were not registered due to technical failure. For the same reasons highlighted in footnote 2 we decided to exclude these six participants only from the analysis of the behavioral response, also considering that the sample size in the behavioral analysis was a less critical issue as we used clearly positive/negative stimuli that are expected to be associated with strong differences and large
} 


\section{Facial EMG}

Facial muscle activation was assessed using EMG measurement over corrugator and zygomaticus. EMG signal in the time window of interest $(-1000 \mathrm{~ms}$ pre-stimulus to $+3000 \mathrm{~ms}$ poststimulus onset) was rectified and root mean square (RMS) transformed. For each trial and muscle, the baseline EMG level was calculated as the mean EMG signal was computed in the $1000 \mathrm{~ms}$ preceding the sentence stimulus. Then, facial EMG response to target stimuli was computed as following. Post-stimulus EMG signal was segmented in 10 time bins of $300 \mathrm{~ms}$ each. The mean signal was computed for each time bin, smoothed across time bins and baseline corrected (i.e. for each trial, muscle and time bin, post-stimulus response was computed as difference relative to the baseline level). We excluded less than $5 \%$ from the analyses using the standard deviation (SD) method (Wilcox, 1992) with the criterion value standing at 3 SDs per muscle. Before statistical analysis, baseline-corrected post-stimulus EMG data were collapsed over the 18 trials with the same emotional expression and linguistic category.

In a preliminary analysis we tested EMG response to neutral fillers: a 2 (Muscle: corrugator, zygomaticus) $\times 2$ (Linguistic category: descriptive action verb, interpretative action verb) $\times 10$ (Time: $1-10)$ repeated measures ANOVA on the mean baseline-corrected rectified EMG signal was performed. This analysis showed only a main effect of Time, $\left(F(9,171)=2.57, p=.009\right.$, Partial $\left.\eta^{2}=.12\right)$, suggesting that in both muscles and for both neutral descriptive action verbs and interpretative action verbs there were unspecific changes in facial reactivity over time, possibly due to task requirements. A series of Dunnet tests comparing post-stimulus time bins against pre-stimulus (baseline) EMG level, showed a small phasic increase in EMG level at time bin 3 (601-900 ms after stimulus onset; $M=.007$ $\mu \mathrm{V}, S D=.01 ; p=.027)$ and $4(901-1200 \mathrm{~ms} ; M=.006 \mu \mathrm{V}, S D=.01 ; p=.063)$. No other 
comparisons approached statistical significance (all -.0007 $\mu \mathrm{V}<M<0.004 \mu \mathrm{V}$; all $p>.21$ ).

To provide a better esteem of facial response to emotional sentences and remove the unspecific changes in facial EMG documented in the preliminary analysis, for each muscle and condition we subtracted the baseline-corrected EMG signal collected during neutral fillers from that recorded during emotional sentences. We then carried out a 2 (Muscle: corrugator, zygomaticus) $\times 2$ (Valence: negative, positive) $\times 2$ (Linguistic category: descriptive action verb, state verb) $\times 10$ (Time: 1-10) repeated measures ANOVA on the mean baseline-corrected rectified EMG signal (time bins of $300 \mathrm{~ms}$; emotional sentences minus neutral fillers). While in the remaining, we focus on this analysis, the same Muscle $\times$ Valence $\times$ Linguistic category $\times$ Time ANOVA performed on baseline-corrected rectified EMG signal with no correction for neutral fillers led to comparable statistical results (not shown here) and main conclusions. Post-hoc analysis was carried out with Duncan test to correct for multiple comparisons. In addition, a series of explorative Dunnett tests were used to test whether and when EMG responses were different from zero (i.e., from the EMG response to neutral fillers).

\section{Results}

\section{Manipulation check}

The Valence $\times$ Linguistic category ANOVA on valence ratings revealed that positive verbs $(M=6.05, S D=0.12)$ were rated as more positive than negative verbs $(M=2.23, S D=$ $0.08)$ as shown by the main effect of Valence $\left(F(1,17)=749.40, p<.001\right.$, Partial $\left.\eta^{2}=.98\right)$. There was a significant Valence $\times$ Linguistic category interaction indicating that valence ratings differed significantly across linguistic category $\left(F(1,17)=70.08, p<.001\right.$, Partial $\eta^{2}$ $=.81)$. Pairwise comparisons (Duncan tests) revealed that positive state verbs $(M=6.45, S D$ $=0.13)$ were rated as more positive than positive descriptive action verbs $(M=2.72, S D=$ $0.12 ; p<.001)$, and negative state verbs $(M=1.74, S D=0.7)$ were rated as more negative 
than negative descriptive action verbs $(M=2.04, S D=0.40 ; p<.001)$. This provides clear evidence that state verbs were perceived as more emotionally valenced than descriptive action verbs.

\section{Evaluative ratings}

Converging evidence supporting the greater emotionality of state verbs were obtained with liking judgments during the task. The Valence $\times$ Linguistic category ANOVA on liking ratings revealed that sentences containing positive verbs were liked significantly more $(M=$ 4.16, $S D=0.10)$ than sentences containing negative verbs $(M=1.82, S D=0.09)$ as shown by the main effect of Valence $\left(F(1,13)=157.30, p<.001\right.$, Partial $\left.\eta^{2}=.92\right)$. There was a significant Valence $\times$ Linguistic category interaction $\left(F(1,13)=21.75, p<.001\right.$, Partial $\eta^{2}=$ $.63)$ indicating that the reported liking of sentences containing positive and negative verbs differed across the linguistic category used to encode them. Specifically, participants reported higher pleasantness ratings for sentences containing positive state verbs $(M=4.46$, $S D=0.29)$ relative to those containing positive descriptive action verbs $(M=3.86, S D=$ $0.59 ; p=.001)$, whereas they reported lower pleasantness ratings for sentences containing negative state verbs $(M=1.61, S D=0.51)$ relative to sentences containing negative descriptive action verbs $(M=2.04, S D=0.40 ; p=.002)$.

\section{Facial Muscular Activation}

\section{Emotionally congruent facial activation}

The Muscle $\times$ Valence $\times$ Linguistic category $\times$ Time ANOVA on facial EMG data showed a significant Muscle $\times$ Valence interaction $\left(F(1,19)=7.13, p=.019\right.$, Partial $\eta^{2}=$ $.27)$, that was qualified by the significant Muscle $\times$ Valence $\times$ Time interaction $(F(9,171)=$ 2.95, $p=.003$, Partial $\left.\eta^{2}=.13\right)$.

As shown in Figure 1, pairwise comparisons revealed that EMG signal of the corrugator was significantly greater for negative verbs than for positive verbs starting from 
time bin 2 to 10 (301-3000 ms, all $p s<.01$; black asterisks in the left panel of Figure 1), whereas no difference was detected at time bin $1(p=.19)$. For the zygomaticus, we found positive verbs to be associated with greater EMG signal than negative verbs. This difference was not significant at time bin 1-3 (1-900 ms, all $p \mathrm{~s}>.12)$, whereas it reached statistical significance at time bin 4-10 (901-3000 ms, all $p \mathrm{~s}<.04$; black asterisks in the right panel of Figure 1) ${ }^{4}$.

Please insert Figure 1 near here

Dunnett tests showed that corrugator EMG signal for negative verbs was marginally greater than zero at time bin $1(1-300 \mathrm{~ms}, p=.07)$ and significantly greater than zero at time bin 2-10 (301-3000 ms, all $p s<.011$; red line and asterisk in the left panel of Figure 1), whereas for positive verbs it was not different from zero at any time bin (all $p s>.64$ ). Zygomaticus EMG signal for positive verbs was significantly greater than zero at time bin 48 (901-2400 ms, all $p$ s $<.04$; blue line and asterisk in the right panel of Figure 1) but not at time bin 1-3 (1-9000 ms, all $p s>.21)$ or time bin 9-10 (2401-3000 ms, all $p \mathrm{~s}>.16)$, whereas for negative verbs it was not different from zero at any time bin (all $p \mathrm{~s}>.13$ ). These findings confirm that corrugator and zygomaticus were selectively activated by emotionally negative and positive verbs, respectively, and this activity was greater than that detected during

\footnotetext{
${ }^{4}$ The same pattern of statistical results was confirmed in a further control analysis in which we transformed EMG data using z-scores. The Muscle $\times$ Valence $\times$ Linguistic category $\times$ Time ANOVA on z-scores confirmed the significance of the Muscle $\times$ Valence $\times$ Time interaction $\left(F(9,171)=3.62, p=.0002\right.$, Partial $\left.\eta^{2}=.16\right)$. Pairwise comparisons confirmed the greater corrugator activity for negative relative to positive verbs at time points 4, 5 and 7-10 (all $p s<.05$ ) and the greater zygomaticus activity for positive relative to negative verbs at time points 7-10 (all $p s<.002$ ).
} 
processing of neutral fillers. See Figure 1 for details.

\section{Influence of linguistic category}

As shown in Figure 2, the Muscle $\times$ Valence $\times$ Linguistic category $\times$ Time ANOVA showed also a significant Linguistic category $\times$ Time interaction $(F(9,171)=5.80, p<.0001$, Partial $\eta^{2}=.23$ ). Pairwise comparisons (Duncan test) indicates that EMG response for state verbs was marginally greater than for descriptive action verbs at time bin 2-3 (301-900 ms, all $p<.07)$, whereas at time bin 5-10 EMG levels showed an opposite trend, with greater response for descriptive relative to state verbs ${ }^{5}$ (i.e., $1201-3000 \mathrm{~ms}$, all $p$ s <.041). No other comparisons approached significance in the remaining time bins (all $p \mathrm{~s}>.25$ ).

Please insert Figure 2 near here

A further series of Dunnett tests showed that EMG activity for state verbs was significantly different from zero (i.e., from EMG activity during neural fillers) at time bin 2-4 (301-1200 ms, all $p$ s <.006), but not in the remaining time bins (all $p$ s <.08). EMG activity for descriptive action verbs was significantly different from zero at time bin 5-10 (1201-3000 $\mathrm{ms}$, all $p \mathrm{~s}<.02)$. See Figure 2 for details.

\section{Testing of linguistic category influence on emotional congruent facial activation}

Linguistic category $\times$ Time interaction was not qualified by higher-order interactions

\footnotetext{
${ }^{5}$ The higher EMG activity for descriptive action relative to state action verbs was confirmed in the analysis on $z$-scores transformed data. The Muscle $\times$ Valence $\times$ Linguistic category $\times$ Time ANOVA showed a trend toward significance for the Linguistic category $\times$ Time interaction $(p=.09)$. EMG activity for action verbs was numerically higher than for state verbs at time point 5-10 and, critically, this difference reached statistical significance at time point 6,7 and $10(p s<.05)$.
} 
involving Valence and Muscle factors, including the 4-way interaction (all $p \mathrm{~s}>.32$ ). This suggests that the two phasic EMG modulations found for state vs. descriptive action verbs (Figure 2) were similar in both muscles and for both positive and negative verbs. By the same token, the Muscle $\times$ Valence $\times$ Time interaction was not qualified by the 4-way interaction, suggesting emotional congruent facial activation were overall similar for the two types of sentences.

Although the 4-way interaction was not significant, we performed three further analyses to directly test the hypothesis of a superiority of descriptive action verb in driving emotionally congruent facial activations. We started by further analyzing the two phasic modulations found for state and descriptive action verbs (Figure 2) with the aim of clarifying the meaning of these apparently unspecific modulations. As detailed in the Supplementary material online, the analyses suggest a possible contribution of emotionally congruent facial activation to the late EMG increase detected for descriptive action verbs (green triangles in Figure 2), but not to the early increase found with emotional state verbs (grey diamonds in Figure 2).

Most, importantly, in a further analysis we directly compared the magnitude of emotionally congruent facial activations for the two types of sentences across time bins (Figure 3). An index representing a muscle-valence congruency effect (congruent musclevalence combinations minus incongruent muscle-valence combinations) was computed for each time bin, separately for emotional state and descriptive action verbs. Figure 3 shows the time course of the congruency effect for state and descriptive action verbs sentences on EMG level. Pairwise comparisons (Duncan test) between state and descriptive action verbs showed that there was a non-significant trend for descriptive action verbs to be associated to greater congruency effect relative state verbs at time bin $7(p=.10)$ and $8(p=.077)$ whereas no similar trend was detected at other time bins (all $p s>.27$ ). Using a more lenient statistical test 
(Fisher LSD) we found a significant difference at time bin 7-8 (all $p$ s <.049) but not at other time points (all $p s>.18)$.

Please insert Figure 3 near here

Thus, although the main ANOVA did not highlight higher-order interactions -and thus we have to be cautious in drawing strong conclusions- the three additional analyses suggested that: i) the early and transient increase in EMG activity for emotional state verbs sentences (Figure 2) reflected an unspecific response that occurred on top of emotion congruent facial activation (see Supplementary material online); ii) emotionally congruent facial emotional reactions partially contributed to the late increase in EMG activity for emotional descriptive action verbs (Figure 2; Supplementary material online); and, critically, iii) relative to state verbs, descriptive action verbs tended to be associated with greater emotionally congruent facial emotional reaction, in particular at time bin 7-8 (1801-2400 ms after stimulus onset).

\section{Discussion and Conclusion}

In the present research we used facial EMG to test corrugator (frowning) and zygomaticus (smiling) activity while participants read and evaluated sentences describing an agent in an emotional state (state verbs) or performing an emotional facial action (descriptive action verbs). We found that these sentences were associated to valence congruent emotional ratings and facial activations. As expected sentences with emotionally positive and negative meanings were rated positively and negatively, respectively. Moreover positive sentences tended to activate the zygomaticus, whereas negative sentences tended to activate the 
corrugator (as shown by the significant Muscle $\times$ Valence $\times$ Time interaction). These findings support the notion that understanding sentences referring to positive and negative emotional concepts is at least partially mediated by an embodied simulation of the linguistic meanings which triggers activity in facial muscles involved in making smiling and frowning expressions, respectively (Barrett, 2006; Barsalou, 2008; Barsalou et al., 2003; de Vega et al., 2008; Foroni \& Semin, 2009, 2013; Niedenthal, 2007; Niedenthal et al., 2009, 2010; Vigliocco et al., 2011).

One major goal of our study was to directly compare emotional congruent facial activation in the two linguistic categories. According to the embodied simulation framework, we hypothesized that understanding state verb sentences would be associated with reactivation of related emotional states in the participant. Based on the Linguistic Category Model (Semin \& Fiedler, 1988; 1991; Rubini et al 2014; Semin \& Marsman, 1994) we expected these state verbs would convey more emotional information. As a consequence of this, we predicted higher emotional ratings for state verbs. Moreover, we expected robust emotionally congruent facial activation, reflecting the somatic correlates of embodied simulation of relatively intense emotions.

On the other hand, understanding more concrete emotional sentences like "Mario smiles" would trigger not only emotion simulation (to some extent) but more simulation of the motor behavior described in the sentence, which would more directly activate congruent facial muscles. Thus, we hypothesized that action verb sentences would induce robust and possibly stronger emotionally congruent facial activation - despite their relatively lower emotional features.

Our experiment provides support to our hypotheses. State verb sentences were rated as more emotional than descriptive action verb sentences, as indicated by both liking and valence judgments. Emotional state verbs (e.g., "Mario enjoys") were perceived in more 
positive terms compared to descriptive action verbs (e.g., "Mario smiles"), possibly because smiling does not necessarily imply actual enjoyment, as in the case of deliberate or social smiles. Similarly, "Mario gets angry" was perceived more negatively compared to "Mario frowns" (frowning does not necessarily imply negative feelings).

Regarding the supposed superiority of descriptive action verb sentences in driving emotionally congruent facial activation, our study provides at least partial support to the hypothesis. On the one hand, both types of sentences induced robust activation of emotionally congruent facial muscles. Moreover, our main analysis suggested that the two linguistic categories induced similar emotionally congruent facial activations, as evidenced by the Muscle $\times$ Valence $\times$ Time interaction, that was not qualified by the 4 -way interaction involving the Linguistic category factor.

On the other hand, we detected differences in the effects triggered by the two linguistic categories. First, the significant Time x Linguistic category interaction clearly revealed distinct time-specific facial responses for sentences including state and descriptive action verbs. In general, emotional state verbs tended to increase the EMG level early in time (301-1200 ms) relative to neutral fillers. Since state verbs were judged with higher emotional ratings, these effects may reflect an unspecific arousal response (i.e., similar in both muscles and for both positive and negative verbs) that occurred on top of the emotionally congruent facial responses (i.e., the activation of zygomaticus and corrugator for positive and negative sentences) (See also Supplemental material online). In contrast, descriptive action verbs tended to increase EMG level at later timing (at about 1501-3000 ms).

Critically, we performed further analyses to directly assess the magnitude of emotionally congruent facial reactions and found evidence that such reactions contributed to the late increase of EMG level detected for descriptive action verbs (Supplemental material online). Even more importantly, direct comparisons between the two linguistic categories 
showed greater emotionally congruent facial reactions for action relative to state verbs in a late time window (i.e., at time bin 7-8, 1801-2400 ms after stimulus onset; see Figure 3). This difference could be clearly observed only when using a lenient statistical threshold.

These results should be interpreted with caution because the 4-way interaction did not reach statistical significance in the main analysis suggesting that that overall state verbs and action verbs tended to elicit similar facial motor reactions. Nevertheless, the weak but noticeable greater responses for descriptive actions verb sentences - despite the low emotional feature of such linguistic stimuli - is well in keeping with embodied theories of action language, emphasizing the action-perception link (e.g., Barsalou, 2008; Gallese, 2008; Fischer \& Zwaan, 2008; Pulvermüller, 2005, 2012) and suggesting that sentences like "Mario smiles" would directly map onto specific facial motor response. This notion has been supported by neuroimaging and neurophysiological studies reporting motor and premotor brain activation during comprehension of action-related language (Candidi, Leone Fernandez, Barber, Carreiras, \& Aglioti, 2010; de Vega et al., 2008; Jirak, Menz, Buccino, Borghi, \& Binkofski, 2010; Moreno, de Vega, Leòn, Bastiaansen, Lewis, \& Magyari, 2015; Vigliocco et al., 2011). In particular, several studies found body-part specific somatotopic activations of motor circuits when people process mouth, hand or foot action verbs presented in isolation or embedded in sentences (Aziz-Zadeh, Wilson, Rizzolatti, \& Iacobonin 2006; Buccino, Riggio, Melli, Binkofski, Gallese, \& Rizzolatti, 2005; Hauk, Johnsrude, \& Pulvermüller, 2004; Kemmerer, Castillo, Talavage, Patterson, \& Wiley, 2008; Tettamanti, Buccino, Saccuman, Gallese, Danna, Scifo, Fazio, et al. 2005). Notably, similar somatotopic motor activations are commonly found when making actions or seeing other people performing the same actions (Avenanti, Bolognini, Maravita, \& Aglioti 2007; Buccino, Binkofski, Fink, Fadiga, Fogassi, Gallese, Seitz, et al., 2001; Caspers, Zilles, Laird, \& Eickhoff, 2010; Rizzolatti, Cattaneo, Fabbri-Destro, \& Rozzi, 2014; Tidoni, Borgomaneri, di Pellegrino, \& Avenanti, 2013; 
Urgesi, Candidi, \& Avenanti, 2014) supporting the notion that understanding action-language is associated with an embodied motor simulation grounded in action-perception systems.

It should be noted that noticeable greater emotionally congruent facial response for action relative to state verb sentences occurred late and in a narrow time window. These data do not provide direct support to the hypothesis of a faster recruitment of motor resources during action-language comprehension, as the "direct mapping" hypothesis would predict. However, they are not incompatible with such possibility. Indeed, such a hypothesis refers to central brain processes. In principle, the late EMG modulations we detected may reflect the somatic consequences of brain events occurring much earlier in time.

Additional factors may explain the weak EMG differences between state and action verbs we detected in our experiment. These weak differences stand in apparent contrast with the clear dissociations reported by Foroni and Semin (2009) who compared action verbs referring to facial expressions with adjectives that are very abstract and had no clear reference to those expressions. However, differently from the stimulus material used by Foroni and Semin (2009), we compared descriptive action verbs with state verbs that are more emotional and retain an indirect link with facial expressions. Indeed, emotional state verbs mainly differ with regard to the level of abstraction in representing such expressions since they do not refer to the action per se but rather the underlying state which is the cause of the expression (Prati, Menegatti, \& Rubini, 2015; Semin \& Fiedler, 1988, 1991).

The active (liking) task may have also contributed to the observed pattern of results. In principle, this task may have masked potentially stronger differences in the facial response to abstract state verbs and concrete descriptive action verbs. Previous EMG studies have shown that some "abstract" words like adjectives, induce robust facial response during semantic tasks (i.e., judging whether the word has an emotional meaning), but little or no facial response during non-semantic perceptual tasks (Niedenthal et al., 2009). Conversely, 
Foroni and Semin (2009) found that action verbs elicited strong facial responses even during passive exposure (Foroni \& Semin, 2009), whereas adjectives activated the same facial responses but weaker in magnitude. Based on these findings, one could suggest that our active task, since it required to evaluate the likability of the sentence (and thus process emotional concepts similarly to the study of Niedenthal et al., 2009), may have also generically increased facial responses to sentences containing both concrete (descriptive action) and abstract (state) verbs and partially masked differences between them.

While future studies are needed to disentangle the relative contribution of task- and stimulus-related factors in driving emotionally congruent facial responses, the Linguistic category $\mathrm{x}$ Time interaction suggest two distinct phasic EMG responses for state and descriptive action verb sentences. This speaks against a generalized masking effect of our task and rather suggests that sentences with more abstract state verbs were processed differently relative to sentences with more concrete descriptive action verbs.

Finally, we should note that, differently from previous EMG works (Foroni \& Semin, 2009, 2013) we investigated third person subject-verb sentences (e.g., "Mario smiles", "Mario is enraged"), which are closer to natural language use in social interaction. Since recent transcranial magnetic stimulation studies reported increased motor-evoked potentials for first person action verb sentences and not for third person action verb sentences (Papeo, CorradiDell'Acqua, \& Rumiati, 2011), Foroni and Semin (2013) speculated a specificity of motor involvement in processing first person and not third person sentences. Our results suggest that this may not be the case, as both state and descriptive action verbs in third person subjectverb sentences induced motor responses in facial muscles (see also, Fourkas, Avenanti, Urgesi, \& Aglioti, 2006; Patuzzo, Fiaschi, \& Manganotti, 2003; Schütz-Bosbach, Avenanti, Aglioti, \& Haggard, 2009 for convergent results in the motor imagery and action observation domain). 
The study has also some limitations concerning our sample that was not large and well-balanced in terms of gender. Although previous research on facial EMG response (Dimberg \& Lundqvist, 1990) have not found many gender differences (e.g., females may show more pronounced, but not qualitatively different emotionally congruent facial effects than male subjects), we cannot exclude that gender may have an impact on facial reactions induced by emotional language processing. Thus, future research should investigate the moderating role of such factors in language triggered facial muscle activation using a larger and more gender balanced sample.

\section{Conclusion}

The present study goes beyond previous studies by examining a wider verb repertoire referring to emotional expressions while more stringently testing the modulating role of linguistic abstraction and emotionality in emotion perception and embodied facial muscular responses to emotional language. Most tellingly, we provide original evidence that language driven facial responses are sensitive also to third person subject-verb sentences composed by linguistic categories at different levels of language abstraction. We found emotional sentences including state and action verb categories to have valence-congruent effects on emotional ratings and corresponding facial muscle activations. Moreover, despite state verbsentences were judged with higher valence ratings than action verbs-sentences, emotional congruent facial activations showed a tendency for greater EMG modulation when reading action relative to state verbs sentences. These results support embodied theories of language comprehension and suggest that understanding emotional action and state verb sentences relies on partially dissociable motor and emotional processes. 
Facial responses to emotional language 26 


\section{Acknowledgements}

The research reported in this article was supported by grants from the 'Excellence Fund' of Albanian Ministry of Education and Science awarded to E.F.; Alma Mater Studiorum, University of Bologna, 219/2012 and MIUR, FIRB "Future in Research" 2012 RBFR128CR6_004 awarded to M.M.; Cogito Foundation (Research project 2013, R-117/13 and Research project 2014, 14-139-R), Ministero Istruzione, Università e Ricerca (Futuro in Ricerca 2012, RBFR12F0BD) and Ministero della Salute (Bando Ricerca Finalizzata Giovani Ricercatori 2010, GR-2010-2319335) awarded to A.A. Special thanks are due to M. Marzocchi for technical support and helping with the script.

\section{Author Contributions}

E.F., M.M., A.A., M.R. designed the experiment; E.F. and M.M. performed the experiment; E.F. filtered and prepared the data; E.F., M.M. and A.A. analyzed data; E.F., M.M., A.A., and M.R. wrote the manuscript. 


\section{References}

Adolphs, R. (2002). Recognizing emotion from facial expressions: Psychological and neurological mechanisms. Behavioral Cognitive Neuroscience Reviews, 1, 21-62. doi:10.1177/1534582302001001003.

Avenanti, A., Bolognini, N., Maravita, A., \& Aglioti, S.M. (2007). Somatic and motor components of action simulation. Current Biology, 17, 2129-2135. doi:10.1016/j.cub.2007.11.045

Avenanti, A., Candidi, M., \& Urgesi, C. (2013). Vicarious motor activation during action perception: beyond correlational evidence. Frontiers in Human Neuroscience, 7, 185. doi: 10.3389/fnhum.2013.00185.

Aziz-Zadeh, L., Wilson, S.M., Rizzolatti, G., Iacoboni, M., 2006b. Congruent Embodied Representations for Visually Presented Actions and Linguistic Phrases Describing Actions. Current Biology, 16, 1818-1823. Doi: 10.1016/j.cub.2006.07.060

Barrett, L. F. (2006). Solving the emotion paradox: categorization and the experience of emotion. Personality and Social Psychology Review, 10, 20-46. doi:10.1207/s15327957pspr1001_2

Barsalou, L.W. (1999). Perceptual symbol systems. Behavioral Brain Science, 22, 577-66.

Barsalou, L.W. (2008). Grounded Cognition. Annual Review of Psychology, 59, 617-645. Doi: 10.1146/annurev.psych.59.103006.093639

Barsalou, L. W., Niedenthal, P. M., Barbey, A., \& Ruppert, J. (2003). Social embodiment. In B. Ross (Ed.), The psychology of learning and motivation (Vol. 43, pp. 43-92). San Diego, CA: Academic Press. 
Bertinetto, P. M., Burani, C., Laudanna, A., Marconi, L., Ratti, D., Rolando, C., \& Thornton A. M. (2005). Corpus e Lessico di Frequenza dell'Italiano Scritto (CoLFIS) [Corpus and Lexicon of Frequences of Written Italian].

Borgomaneri, S., Gazzola, V., \& Avenanti, A. (2014a). Temporal dynamics of motor cortex excitability during perception of natural emotional scenes. Social Cognitive and Affective Neuroscience, 9, 1451-7. Doi: 10.1093/scan/nst139.

Borgomaneri, S., Gazzola, V., \& Avenanti, A. (2014b). Transcranial magnetic stimulation reveals two functionally distinct stages of motor cortex involvement during perception of emotional body language. Brain Structure and Function. Jul 152014 [Epub ahead of print] Doi: 10.1007/s00429-014-0825-6.

Boulenger, V., Hauk, O., Pulvermüller, F. (2009), Grasping ideas with the motor system: semantic somatotopy in idiom comprehension, Cerebral Cortex, 19, 1905-14. Doi:10.1093/cercor/bhn217

Brambilla, M., Sacchi, S., Menegatti, M., \& Moscatelli, S. (2016). Honesty and dishonesty don't move together: Trait content information influences behavioral synchrony. Journal of Nonverbal Behavior, published online ahead of print. Doi: 10.1007/s10919-0160229-9

Buccino, G., Binkofski, F., Fink, G. R., Fadiga, L., Fogassi, L., Gallese, V., Seitz, R. J., Zilles, K., Rizzolatti, G., Freund, H. J. (2001). Action observation activates premotor and parietal areas in a somatotopic manner: an fMRI study. Europena Journal of Neuroscience, 13, 400-404. Doi: 10.1046/j.1460-9568.2001.01385.x

Buccino G., Riggio L., Melli G., Binkofski F., Gallese V., \& Rizzolatti G. (2005). Listening to action-related sentences modulates the activity of the motor system: a combined TMS and behavioural study. Cognitive Brain Research, 24, 355-363.

Doi:10.1016/j.cogbrainres.2005.02.020 
Candidi, M., Leone Fernandez, B., Barber, H., Carreiras, M., \& Aglioti, S. M. (2010). Hands on the future: facilitation of cortico-spinal hand-representation when reading the future tense of hand-related action ve 1 rbs. European Journal Of Neuroscience, 32 , 677-683. doi: 10.1111/j.1460-9568.2010.07305.x

Caspers, S., Zilles, K., Laird, A. R., \& Eickhoff, S. B. (2010). ALE meta-analysis of action observation and imitation in the human brain. Neuroimage, 50, 1148-1167. Doi: 10.1016/j.neuroimage.2009.12.112

Chartrand, T. L., Maddux, W. W., \& Lakin, J. L. (2005). Beyond the perception-behavior link: The ubiquitous utility and motivational moderators of non-conscious mimicry. In R. R. Hassin, J. S. Uleman, \& J. A. Bargh (Eds.), The new unconscious (pp. 334-361). New York: Oxford University Press. Doi: 10.1007/s10579-007-9057-1.

Chartrand, T. L., \& Van Baaren, R. B. (2009). Human mimicry. In M. P. Zanna (Ed.), Advances in Experimental Social Psychology (pp. 219-274). San Diego (CA): Academic Press.

Coenen, L. H. M., Hedebouw, L., \& Semin, G. R. (2006). Measuring Language Abstraction: The Linguistic Category Model (LCM). Retrieved from http://cratylus.org/resources/linguistic-model

De Vega, M., Glenberg, A., \& Graesser, A., 2008. Symbols and Embodiment: Debates on Meaning and Cognition. Oxford University Press, New York, pp. 397-440.

Delplanque, S., Grandjean, D., Chrea, C., Coppin, G., Aymard L., Cayeux, I., et al. (2009). Sequential unfolding of novelty and pleasantness appraisals of odors: Evidence from facial electromyography and autonomic reactions. Emotion, 9, 316-328.

Doi:10.1037/a0015369 
Dimberg, U. \& Petterson, M. (2000). Facial reactions to happy and angry facial expressions: Evidence for right hemisphere dominance. Psychophysiology, 37, 693-696. Doi:10.1017/S0048577200990759

Dimberg, U. \& Lundqvist, L. O. (1990). Gender differences in facial reactions to facial expressions. 1 Biological Psychology, 30, 151-159. Doi: 10.1016/03010511(90)90024-

Dimberg, U., Thunberg, M., \& Elmehed, K. (2000). Unconscious facial reactions to emotional facial expressions. Psychological Science, 11, 86-89. Doi: 10.1111/14679280.00221

Fischer, M. \& Zwaan, R. (2008). Embodied Language: A Review of the Role of the Motor System in Language Comprehension. The Quarterly Journal of Experimental Psychology, 61, 825-850. Doi: 10.1080/17470210701623605

Foroni, F. \& Semin, G.R. (2009). Language that puts you in touch with your bodily feelings: The Multimodal. Responsiveness of Affective Expressions. Psychological Science, 20,974-980. Doi:10.1111/j.1467-9280.2009.02400.x

Foroni, F. \& Semin, G. R. (2013). Comprehension of action negation involves inhibitory simulation. Frontiers in Human Neuroscience, 30 May 2013.

Doi:10.3389/fnhum.2013.00209

Fourkas, A. D., Avenanti, A., Urgesi, C., \& Aglioti, S. M. (2006). Corticospinal facilitation during first and third person imagery. Experimental Brain Research, 168, 143-51. Doi:10.1007/s00221-005-0076-0

Friedlung, A. J. \& Cacioppo, J. T. (1989). Guidelines for Human Electromyographic Research. Psychophysiology, 23, 567 - 586. Doi: 10.1111/j.14698986.1986.tb00676.x 
Gallese, V. (2008). Mirror neurons and the social nature of language: The neural exploitation hypothesis. Social Neuroscience, 3, 317-333.

Glenberg, A. M. (2007). Language and action: creating sensible combination of ideas. In G. Gaskell (Ed.), Handbook of psycholinguistics (pp. 361-371). Oxford (UK): Oxford University Press.

Glenberg, A. M. \& Gallese, V. (2012). Action-1 based language: A theory of language acquisition, comprehension, and production. Cortex, 1-18. doi:10.1016/j.cortex.2011.04.010.

Glenberg, A.M., Havas, D., Becker, R., \& Rinck, M. (2005). Grounding Language in Bodily States: The Case for Emotion. In R. Zwaan \& D. Pecher (Eds.), The grounding of cognition: The role of perception and action in memory, language, and thinking (pp.115-128). Cambridge: Cambridge University Press.

Goldman, A. I. \& Sripada, C. S. (2005) Simulationist models of face-based emotion recognition. Cognition, 94, 193-213. Doi: 10.1016/j.cognition.2004.01.005

Hauk, O., Johnsrude, I., Pulvermüller, F. (2004). Somatotopic Representation Of Action Words In Human Motor And Premotor Cortex. Neuron, 41, 301-307. Doi:10.1016/S0896- 273(03)00838-9

Jirak, D., Menz, M. M., Buccino, G., Borghi, A. M., \& Binkofski, F. (2010). Grasping language - a short story on embodiment. Conscious Cognition, 19, 711-20. doi:10.1016/j.concog.2010.06.020.

Kemmerer, D., Castillo, J. G., Talavage, T., Patterson, S., \& Wiley, C. (2008). Neuroanatomical distribution of five semantic components of verbs: evidence from fMRI. Brain Language, 107, 16 - 43. Doi: 10.1016/j.bandl.2007.09.003 
Keysers, C., \& Gazzola, V. (2009). Expanding the mirror: vicarious activity for actions, emotions, and sensations. Current Opinion in Neurobiology 19, 666-67. Doi:10.1016/j.conb.2009.10.006

Larsen, J. T., Norris, C. J., \& Cacioppo, J.T., (2003). Effects of positive and negative affect on electromyographic activity over zygomaticus major and corrugator supercilii. Psychophysiology, 40, 776-785. Doi: 10.1111/1469-8986.00078

Menegatti, M., \& Rubini, M. (2014). Initiating, maintaining, or breaking up? The motivated use of language abstraction in romantic relationships. Social Psychology, 45, 408420. Doi: $10.1027 / 1864-9335 / a 000200$

Moreno, I., de Vega, M., Leòn, I., Bastiaansen, M., Lewis, A. G., \& Magyari (2015). Brain dynamics in the comprehension of action-related language. A time-frequency analysis of mu rhythms. Neuroimage, 109, 50-62. doi: 10.1016/j.neuroimage.2015.01.018.

Niedenthal, P. M. (2007). Embodying emotion. Science, 316, 1002-1005. Doi:10.1126/science. 1136930

Niedenthal, P. M., Mermillod, M., Maringer, M., \& Hess, U. (2010). The Simulation of Smiles (SIMS) model: Embodied simulation and the meaning of facial expression. Target article for Behavioral and Brain Sciences, 33, 417-480.

Doi:10.1017/S0140525X10000865

Niedenthal, P. M., Winkielman, P., Mondillon, L., \& Vermeulen, N. (2009). Embodiment of emotion concepts. Journal of Personality and Social Psychology, 96, 120-136. Doi:10.1037/a0015574

Papeo, L., Corradi-Dell'Acqua, C., \& Rumiati, R.I. (2011). "She" is not like "I": The tie between language and action is in our imagination. Journal of Cognitive Neuroscience, 12, 3939-48. Doi: 10.1162/jocn_a_00075 
Patuzzo, S., Fiaschi, A., \& Manganotti, P. (2003). Modulation of motor cortex excitability in the left hemisphere during action observation: a single- and paired-pulse transcranial magnetic stimulation study of self- and non-self-action observation. Neuropsychologia, 41, 1272-8. Doi: 10.1016/S0028-3932(02)00293-2

Pecher, D., Zeelenberg, R., \& Barsalou, L.W. (2004). Sensorimotor simulations underlie conceptual representations: Modality-specific effects of prior activation. Psychonomic Bulletin \& Review, 11, 164-167. Doi: 10.3758/BF03206477

Prati, F., Menegatti, M., \& Rubini, M. (2015). The Beneficial Role of Multiple Categorization and Intergroup Contact in Reducing Linguistic Outgroup Derogation. Journal of Language and Social Psychology, 34, 475-500. Doi:10.1177/0261927X14567777

Pulvermüller, F. (1999). Words in the brain's language. Behavoral Brain Science, 22, 253-36. Doi: 10.1016/j.bandl.2004.12.007

Pulvermüller, F. (2005). Brain mechanisms linking language and action. Nature Review Neuroscience, 6, 576-582. Doi: 10.1038/nrn1706

Pulvermüller, F. (2012). Meaning and the brain: The neurosemantics of referential, interactive, and combinatorial knowledge. Journal of Neurolinguistics, 25, 423-459. doi: 10.1016/j.jneuroling.2011.03.004

Rizzolatti, G., Cattaneo, L., Fabbri-Destro, M., \& Rozzi, S. (2014). Cortical mechanisms underlying the organization of goal-directed actions and mirror neuron-based action understanding. Physiological Review, 94, 655-706. doi: 10.1152/physrev.00009.2013.

Rubini, M., Menegatti, M., \& Moscatelli, S. (2014). The strategic role of language abstraction in achieving symbolic and practical goals. European Review of Social Psychology, 25, 263-313. Doi: 10.1080/10463283.2014.985501 
Schütz-Bosbach, S., Avenanti, A., Aglioti, S. M., \& Haggard, P. (2009). Don't do it! Cortical inhibition and self-attribution during action observation. Journal of Cognitive Neuroscience, 21, 1215-1227. Doi: 10.1162/jocn.2009.21068.

Semin, G. R. \& Fiedler, K (1988). The Cognitive Functions of Linguistic Categories in Describing Persons' Social Cognition and Language. Journal of Personality and Social Psychology, 54, 558-568. Doi:10.1037/0022-3514.54.4.558

Semin, G.R., \& Fiedler, K. (1991). The linguistic category model, its bases, applications, and range. In W. Stroebe \& M. Hewstone (Eds.), European review of social psychology (Vol. 2, pp. 1-30). Chicherster: Wiley.

Semin, G. R., \& Fiedler, K. (1 1992). The inferential properties of interpersonal verbs. In G. R. Semin and K. Fiedler (Eds.), Language, interaction and social cognition (pp. 5878). Newbury Park, CA: Sage.

Semin, G. R. \& Marshman, J. G (1994). “Multiple inference-inviting properties” of interpersonal verbs: Event istigation, dispositional inference and implicit causality. Journal of Personality and Social Psychology, 67, 836-849. Doi: 10.1037/00223514.67.5.836

Tettamanti, M., Buccino, G., Saccuman, M. C., Gallese, V., Danna, M., Scifo, P., Fazio, F., Rizzolatti, G., Cappa, S. F., \& Perani D. (2005). Listening to action-related sentences activates fronto-parietal motor circuits. Journal of Cognitive Neuroscience, 17, 273 281. Doi: 10.1016/j.cogbrainres.2005.02.020

Tidoni, E., Borgomaneri, S., di Pellegrino, G. \& Avenanti, A. (2013). Action simulation plays a critical role in deceptive action recognition. The Journal of Neuroscience, 33, 611623.doi: 10.1523/JNEUROSCI.2228-11.2013

Urgesi, C., Candidi, M., \& Avenanti, A. (2014). Neuroanatomical substrates of action perception and understanding: an anatomic likelihood estimation meta-analysis of 
lesion-symptom mapping studies in brain injured patients. Frontiers of Human Neuroscience, 8, 344. doi: 10.3389/fnhum.2014.00344

Vigliocco, G., Vinson, D. P., Druks, J., Barber, H., \& Cappa, S. F. (2011). Nouns and verbs in the brain: a review of behavioural, electrophysiological, neuropsychological and imaging studies. Neuroscience \& Biobehavoral Reviews, 35, 407-426.

Doi:10.1016/j.neubiorev.2010.04.007

23 Vrana, S. R. (1993). The psychophysiology of disgust: Differentiating negative emotional 24 contexts with facial EMG. Psychophysiology, 30, 279-286.

Doi:10.1111/j.14698986.1993.tb03354.x

Vrana, S. R., \& Gross, D. (2004). Reactions t 1 o facial expressions: Effects of neutral expressions, gender, speech anxiety. Biological Psychology, 66, 63-78. doi:10.1016/j.biopsycho.2003.07.004

Vrana, S. R., \& Rollock, D. (2002). The role of ethnicity, gender, emotional content and contextual differences in physiological, expressive, and self-reported emotional responses to imagery. Cognition and Emotion, 16, 165-192.

Doi:10.1080/02699930143000185

Willems, R. M., Toni, I., Hagoort, P., \& Casasanto, D. (2009). Body-specific motor imagery of hand actions: Neural evidence from right- and left-handers. Frontiers in Human Neuroscience, 3, 1-9. Doi: 10.3389/neuro.09.039.2009 


\section{Figure legends}
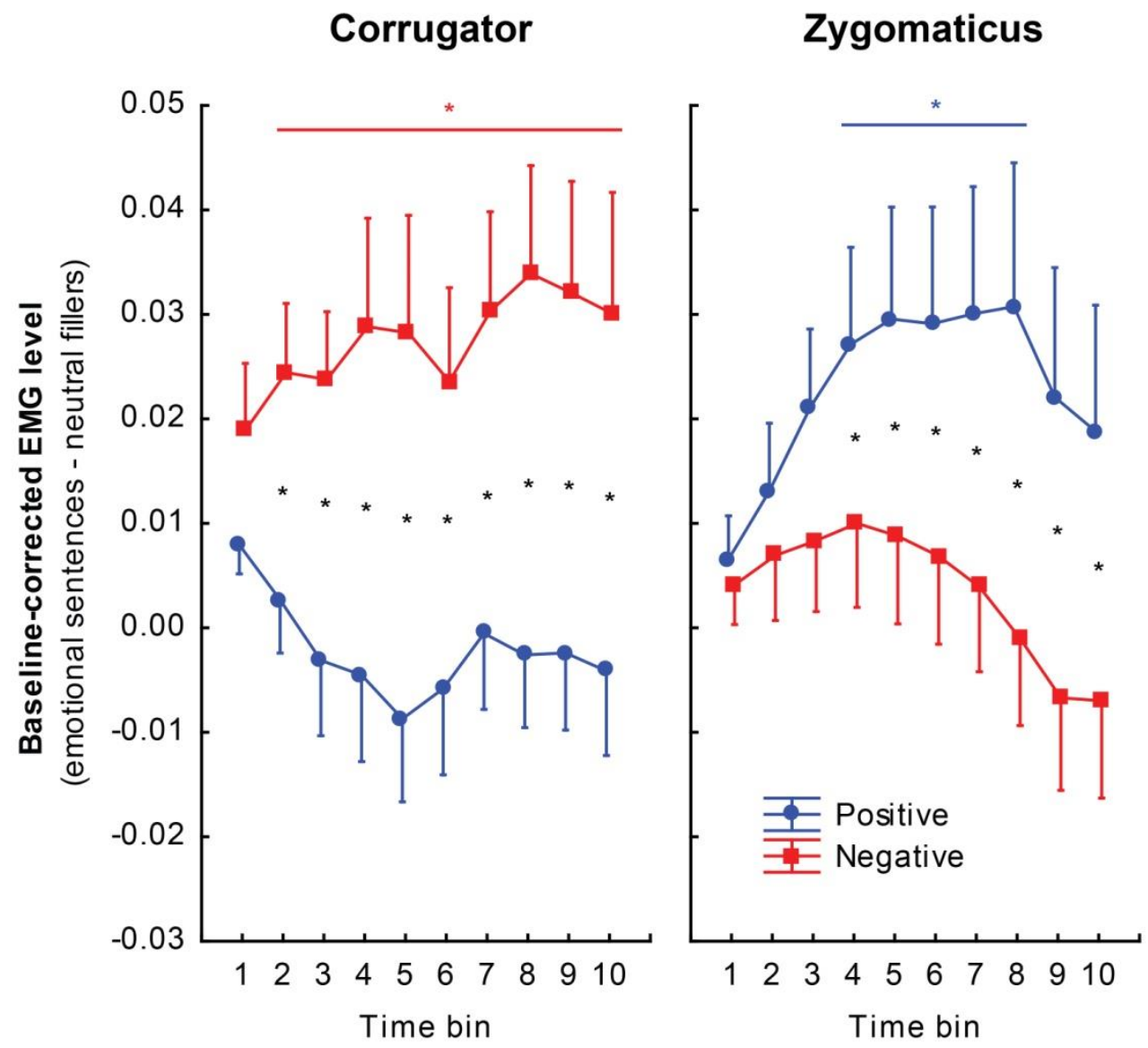

Figure 1. Muscle $\times$ Valence $\times$ Time interaction showing the emergence of facial reactions in the Corrugator (left panel) and Zygomaticus (right panel) in microvolt. The EMG baseline corrected response to positive and negative verbs minus the response to neutral fillers is represented in blue circles and red squares, respectively. Data from action and state verbs are averaged. Blue and red asterisks (*) indicate significant difference relative to baseline. Black asterisks $(*)$ indicate comparisons between positive and negative verbs. Error bars denote s.e.m. 


\section{Corrugator \& Zygomaticus}

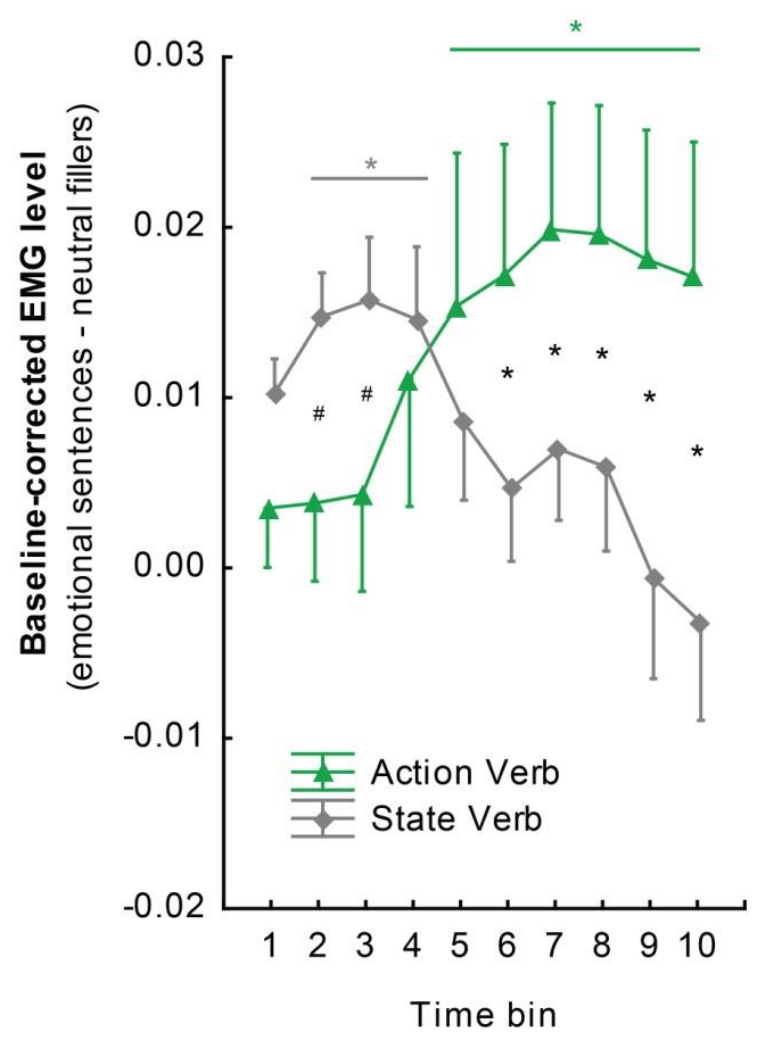

Figure 2. Verb x Time interaction showing an early increase of EMG response in microvolt for state relative to descriptive action verbs and a later opposite trend. EMG response to Action and State verbs is represented with green triangles and grey diamonds, respectively. Data from zygomaticus and corrugator and from positive and negative sentences are averaged. Green and grey symbols indicate significant $(*)$ or marginally significant (\#) difference relative to baseline. Black symbols indicate comparisons between descriptive and state verbs. Error bars indicate s.e.m. 


\section{EMG Congruency effect}

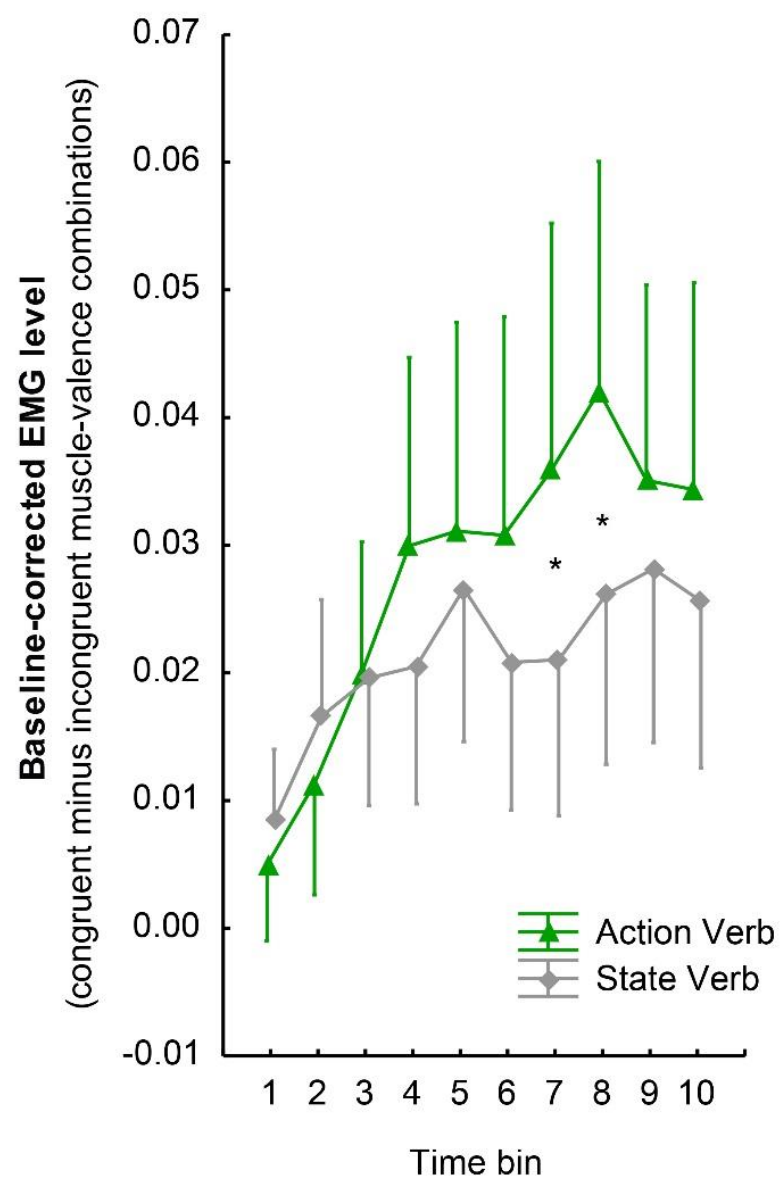

Figure 3. Congruency effect on facial muscles. EMG response to descriptive action and state verbs is represented with green triangles and grey diamonds, respectively. For each time bin, data from zygomaticus and corrugator during processing of positive and negative sentences (i.e., congruent muscle-valence combinations) are averaged and contrasted with data from zygomaticus and corrugator during processing of negative and positive sentences (i.e., incongruent muscle-valence combinations). Black asterisks (*) indicate significant comparisons between sentences containing descriptive and state verbs. Error bars denote s.e.m. 


\section{Supplementary material online}

As reported in the main text, the Linguistic category $\mathrm{x}$ Time interaction was not qualified by higher-order interactions involving Valence and Muscle factors, including the 4way interaction (all $p \mathrm{~s}>.32$ ). This suggests that the two phasic EMG modulations found for state vs. descriptive action verbs (Figure 2) - that occurred on top of the emotionally congruent facial activations - were similar in both muscles and for both positive and negative verbs. While the pattern of results is suggestive of (muscle- and valence-unspecific) arousal responses that add to the emotion congruent facial activation, we performed further analyses to investigate the physiological meaning of these responses. Two groups of analyses were conducted separately for state and descriptive action verbs.

\section{EMG response to state verbs}

For emotional state verbs, we considered the time window in which these verbs tended to show greater EMG activity relative to action verbs and neutral fillers i.e. time bin 23 (301-900 ms after stimulus onset, second and third grey diamonds in Figure 2) and computed the mean EMG signal from that window. We first tested whether the increase in the mean EMG level for emotional verbs was greater than for neutral fillers in both muscles. Two $t$-tests confirmed that a significant increase could be detected in both the corrugator $(M$ $=0.018 \mu \mathrm{V}, S D=0.02 ; t(19)=4.08, p<.001)$ and zygomatic muscle $(M=0.018 \mu \mathrm{V}, S D=$ $0.02 ; t(19)=3.39, p=.003)$, suggesting the increase was not carried out by only one of the two muscles or by their average (as also suggested by the lack of interaction with factor Muscle in the main ANOVA).

We then tested the contribution of emotionally congruent facial activations to the EMG response to state verbs. In particular, we tested whether a congruency effect could be detected, by contrasting mean EMG activity for congruent muscle-valence combinations (i.e., 
zygomatic activity for positive state verbs and corrugator activity for negative state verbs) to incongruent muscle-valence combinations (i.e., zygomatic activity for negative state verbs and corrugator activity for positive state verbs). A $t$-test showed no significant difference in EMG level between the two combinations (congruent combination: $M=0.026 \mu \mathrm{V}, S D=$ 0.03; incongruent combination: $M=0.026 \mu \mathrm{V}, S D=0.03 ; t(19)=1.81, p=.09$ ), indicating that for state verbs, no significant congruency effect could be detected in the early time window. These analyses further suggest that the initial increase of EMG activity for emotional vs. neutral state verbs detected at time bin 2-3 reflected a generalized and unspecific response that could be detected in both muscles.

\section{EMG response to action verbs}

In a second group of analyses, we focused on action verbs. We considered the time window at which these verbs showed greater EMG activity relative to state verbs and neutral fillers, that is time bin 6-10 (1501-3000 ms after stimulus onset, $6^{\text {th }}-10^{\text {th }}$ green triangles in Figure 2) and computed the mean EMG signal from that window. An increase in the mean EMG level for action verbs relative to fillers could be detected in both the corrugator $(M=$ $0.022 \mu \mathrm{V}, S D=0.04 ; t(19)=2.52, p=.021)$ and zygomatic muscle $(M=0.046 \mu \mathrm{V}, S D=$ $0.09 ; t(19)=2.19, p=.041)$.

Importantly, also for action verbs, we tested the contribution of emotionally congruent facial activation by investigating whether a congruency effect could be detected. A $t$-test revealed larger EMG activity for congruent muscle-valence combinations (i.e., zygomatic activity for cheek-related action verbs and corrugator activity for brow-related action verbs; $M=0.039 \mu \mathrm{V}, S D=0.07)$ compared to incongruent muscle-valence combinations (i.e., zygomatic activity for corrugator-related action verbs and corrugator activity for zygomaticrelated action verbs; $M=0.003 \mu \mathrm{V}, S D=0.02 ; t(19)=2.11, p=.048)$. This suggests that 
Facial responses to emotional language 42

emotionally congruent facial reactions may have contributed to the apparently unspecific EMG increase detected for action verbs in the 1501-3000 ms time window. 